Proceedings of the International Congress of Mathematicians

Hyderabad, India, 2010

\title{
Non-asymptotic theory of random matrices: extreme singular values
}

\author{
Mark Rudelson, Roman Vershynin ${ }^{\dagger}$
}

\begin{abstract}
The classical random matrix theory is mostly focused on asymptotic spectral properties of random matrices as their dimensions grow to infinity. At the same time many recent applications from convex geometry to functional analysis to information theory operate with random matrices in fixed dimensions. This survey addresses the non-asymptotic theory of extreme singular values of random matrices with independent entries. We focus on recently developed geometric methods for estimating the hard edge of random matrices (the smallest singular value).
\end{abstract}

Mathematics Subject Classification (2000). Primary 60B20; Secondary 46B09

Keywords. Random matrices, singular values, hard edge, Littlewood-Offord problem, small ball probability

\section{Asymptotic and non-asymptotic problems on ran- dom matrices}

Since its inception, random matrix theory has been mostly preoccupied with asymptotic properties of random matrices as their dimensions grow to infinity. A foundational example of this nature is Wigner's semicircle law [96. It applies to a family of $n \times n$ symmetric matrices $A_{n}$ whose entries on and above the diagonal are independent standard normal random variables. In the limit as the dimension $n$ grows to infinity, the spectrum of the normalized matrices $\frac{1}{\sqrt{n}} A_{n}$ is distributed according to the semicircle law with density $\frac{1}{2 \pi} \sqrt{4-x^{2}}$ supported on the interval $[-2,2]$. Precisely, if we denote by $S_{n}(z)$ the number of eigenvalues of $\frac{1}{\sqrt{n}} A_{n}$ that are smaller than $z$, then for every $z \in \mathbb{R}$ one has

$$
\frac{S_{n}(z)}{n} \rightarrow \frac{1}{2 \pi} \int_{-\infty}^{z}\left(4-x^{2}\right)_{+}^{1 / 2} d x \quad \text { almost surely as } n \rightarrow \infty .
$$

In a similar way, Marchenko-Pastur law [55] governs the limiting spectrum of $n \times n$ Wishart matrices $W_{N, n}=A^{*} A$, where $A=A_{N, n}$ is an $N \times n$ random Gaussian matrix whose entries are independent standard normal random variables. As

\footnotetext{
*Partially supported by NSF grant DMS FRG 0652684

${ }^{\dagger}$ Partially supported by NSF grant DMS FRG 0918623
} 
the dimensions $N, n$ grow to infinity while the aspect ratio $n / N$ converges to a non-random number $y \in(0,1]$, the spectrum of the normalized Wishart matrices $\frac{1}{N} W_{N, n}$ is distributed according to the Marchenko-Pastur law with density $\frac{1}{2 \pi x y} \sqrt{(b-x)(x-a)}$ supported on $[a, b]$ where $a=(1-\sqrt{y})^{2}, b=(1+\sqrt{y})^{2}$. The meaning of the convergence is similar to the one in Wigner's semicircle law.

It is widely believed that phenomena typically observed in asymptotic random matrix theory are universal, that is independent of the particular distribution of the entries of random matrices. By analogy with classical probability, when we work with independent standard normal random variables $Z_{i}$, we know that their normalized sum $S_{n}=\frac{1}{\sqrt{n}} \sum_{i=1}^{n} Z_{i}$ is again a standard normal random variable. This simple but useful fact becomes significantly more useful when we learn that it is asymptotically universal. Indeed, The Central Limit Theorem states that if instead of normal distribution $Z_{i}$ have general identical distribution with zero mean and unit variance, the normalized sum $S_{n}$ will still converge (in distribution) to the standard normal random variable as $n \rightarrow \infty$. In random matrix theory, universality has been established for many results. In particular, Wigner's semicircle law and Marchenko-Pastur law are known to be universal - like the Central Limit Theorem, they hold for arbitrary distribution of entries with zero mean and unit variance (see 60, 6, for semi-circle law and 65, 5, for Marchenko-Pastur law).

Asymptotic random matrix theory offers remarkably precise predictions as dimension grows to infinity. At the same time, sharpness at infinity is often counterweighted by lack of understanding of what happens in finite dimensions. Let us briefly return to the analogy with the Central Limit Theorem. One often needs to estimate the sum of independent random variables $S_{n}$ with fixed number of terms $n$ rather than in the limit $n \rightarrow \infty$. In this situation one may turn to BerryEsseen's theorem which quantifies deviations of the distribution of $S_{n}$ from that of the standard normal random variable $Z$. In particular, if $\mathbb{E}\left|Z_{1}\right|^{3}=M<\infty$ then

$$
\left|\mathbb{P}\left(S_{n} \leq z\right)-\mathbb{P}(Z \leq z)\right| \leq \frac{C}{1+|z|^{3}} \cdot \frac{M}{\sqrt{n}}, \quad z \in \mathbb{R}
$$

where $C$ is an absolute constant [11, 23]. Notwithstanding the optimality of BerryEsseen inequality (1.1), one can still hope for something better than the polynomial bound on the probability, especially in view of the super-exponential tail of the limiting normal distribution: $\mathbb{P}(|Z|>z) \lesssim \exp \left(-z^{2} / 2\right)$. Better estimates would indeed emerge in the form of exponential deviation inequalities [61, 47, but this would only happen when we drop explicit comparisons to the limiting distribution and study the tails of $S_{n}$ by themselves. In the simplest case, when $Z_{i}$ are i.i.d. mean zero random variables bounded in absolute value by 1 , one has

$$
\mathbb{P}\left(\left|S_{n}\right|>z\right) \leq 2 \exp \left(-c z^{2}\right), \quad z \geq 0,
$$

where $c$ is a positive absolute constant. Such exponential deviation inequalities, which are extremely useful in a number of applications, are non-asymptotic results whose asymptotic prototype is the Central Limit Theorem.

A similar non-asymptotic viewpoint can be adopted in random matrix theory. One would then study spectral properties of random matrices of fixed dimensions. 
Non-asymptotic results on random matrices are in demand in a number of today's applications that operate in high but fixed dimensions. This usually happens in statistics where one analyzes data sets with a large but fixed number of parameters, in geometric functional analysis where one works with random operators on finitedimensional spaces (whose dimensions are large but fixed), in signal processing where the signal is randomly sampled in many but fixed number of points, and in various other areas of science and engineering.

This survey is mainly focused on the non-asymptotic theory of the extreme singular values of random matrices (equivalently, the extreme eigenvalues of sample covariance matrices) where significant progress was made recently. In Section 2 we review estimates on the largest singular value (the soft edge). The more difficult problem of estimating the smallest singular value (the hard edge) is discussed in Section 3, and its connection with the Littlewood-Offord problem in additive combinatorics is the content of Section 4. In Section 5 we discuss several applications of non-asymptotic results to the circular law in asymptotic random matrix theory, to restricted isometries in compressed sensing, and to Kashin's subspaces in geometric functional analysis.

This paper is by no means a comprehensive survey of the area but rather a tutorial. Sketches of some arguments are included in order to give the reader a flavor of non-asymptotic methods. To do this more effectively, we state most theorems in simplified form (e.g. always over the field $\mathbb{R}$ ); the reader will find full statements in the original papers. Also, we had to completely omit several important directions. These include random symmetric matrices which were the subject of the recent survey by Ledoux 48, and random matrices with independent columns, see in particular [1, 94]. The reader is also encouraged to look at the comprehensive survey [19] on some geometric aspects of random matrix theory.

\section{Extreme singular values}

Geometric nature of extreme singular values The non-asymptotic viewpoint in random matrix theory is largely motivated by geometric problems in high dimensional Euclidean spaces. When we view an $N \times n$ matrix $A$ as a linear operator $\mathbb{R}^{n} \rightarrow \mathbb{R}^{N}$, we may want first of all to control its magnitude by placing useful upper and lower bounds on $A$. Such bounds are conveniently provided by the smallest and largest singular values of $A$ denoted $s_{\min }(A)$ and $s_{\max }(A)$; recall that the singular values are by definition the eigenvalues of $|A|=\sqrt{A^{*} A}$.

The geometric meaning of the extreme singular values can be clear by considering the best possible factors $m$ and $M$ in the two-sided inequality

$$
m\|x\|_{2} \leq\|A x\|_{2} \leq M\|x\|_{2} \quad \text { for all } x \in \mathbb{R}^{n} .
$$

The largest $m$ and the smallest $M$ are precisely the extreme singular values $s_{\min }(A)$ and $s_{\max }(A)$ respectively. They control the distortion of the Euclidean geometry under the action of the linear transformation $A$; the distance between any two points in $\mathbb{R}^{n}$ can increase by at most the factor $s_{\max }(A)$ and decrease by at most 
the factor $s_{\max }(A)$. The extreme singular values are clearly related to the operator norms of the linear operators $A$ and $A^{-1}$ acting between Euclidean spaces: $s_{\max }(A)=\|A\|$ and if $A$ is invertible then $s_{\min }(A)=1 /\left\|A^{-1}\right\|$.

Understanding the behavior of extreme singular values of random matrices is needed in many applications. In numerical linear algebra, the condition number $\kappa(A)=s_{\max }(A) / s_{\min }(A)$ often serves as a measure of stability of matrix algorithms. Geometric functional analysis employs probabilistic constructions of linear operators as random matrices, and the success of these constructions often depends on good bounds on the norms of these operators and their inverses. Applications of different nature arise in statistics from the analysis of sample covariance matrices $A^{*} A$, where the rows of $A$ are formed by $N$ independent samples of some unknown distribution in $\mathbb{R}^{n}$. Some other applications are discussed in Section 5

Asymptotic behavior of extreme singular values We first turn to the asymptotic theory for the extreme singular values of random matrices with independent entries (and with zero mean and unit variance for normalization purposes). From Marchenko-Pastur law we know that most singular values of such random $N \times n$ matrix $A$ lie in the interval $[\sqrt{N}-\sqrt{n}, \sqrt{N}+\sqrt{n}]$. Under mild additional assumptions, it is actually true that all singular values lie there, so that asymptotically we have

$$
s_{\min }(A) \sim \sqrt{N}-\sqrt{n}, \quad s_{\max }(A) \sim \sqrt{N}+\sqrt{n} .
$$

This fact is universal and it holds for general distributions. This was established for $s_{\max }(A)$ by Geman [29] and Yin, Bai and Krishnaiah [97]. For $s_{\min }(A)$, Silverstein 71] proved this for Gaussian random matrices, and Bai and Yin [8] gave a unified treatment of both extreme singular values for general distributions:

Theorem 2.1 (Convergence of extreme singular values, see [8]). Let $A=A_{N, n}$ be an $N \times n$ random matrix whose entries are independent copies of some random variable with zero mean, unit variance, and finite fourth moment. Suppose that the dimensions $N$ and $n$ grow to infinity while the aspect ratio $n / N$ converges to some number $y \in(0,1]$. Then

$$
\frac{1}{\sqrt{N}} s_{\min }(A) \rightarrow 1-\sqrt{y}, \quad \frac{1}{\sqrt{N}} s_{\max }(A) \rightarrow 1+\sqrt{y} \quad \text { almost surely. }
$$

Moreover, without the fourth moment assumption the sequence $\frac{1}{\sqrt{N}} s_{\max }(A)$ is almost surely unbounded [7].

The limiting distribution of the extreme singular values is known and universal. It is given by the Tracy-Widom law whose cumulative distribution function is

$$
F_{1}(x)=\exp \left(-\int_{x}^{\infty}\left[u(s)+(s-x) u^{2}(s)\right] d s\right)
$$

where $u(s)$ is the solution to the Painlevè II equation $u^{\prime \prime}=2 u^{3}+s u$ with the asymptotic $u(s) \sim \frac{1}{2 \sqrt{\pi} s^{1 / 4}} \exp \left(-\frac{2}{3} s^{3 / 2}\right)$ as $s \rightarrow \infty$. The occurrence of TracyWidom law in random matrix theory and several other areas was the subject of 
an ICM 2002 talk of Tracy and Widom [91. This law was initially discovered for the largest eigenvalue of a Gaussian symmetric matrix [89, 90]. For the largest singular values of random matrices with independent entries it was established by Johansson [37] and Johnstone [39] in the Gaussian case, and by Soshnihikov 74] for more general distributions. For the smallest singular value, the corresponding result was recently obtained in a recent work Feldheim and Sodin 25] who gave a unified treatment of both extreme singular values. These results are known under a somewhat stronger subgaussian moment assumption on the entries $a_{i j}$ of $A$, which requires their distribution to decay as fast as the normal random variable:

Definition 2.2 (Subgaussian random variables). A random variable $X$ is subgaussian if there exists $K>0$ called the subgaussian moment of $X$ such that

$$
\mathbb{P}(|X|>t) \leq 2 e^{-t^{2} / K^{2}} \text { for } t>0 .
$$

Examples of subgaussian random variables include normal random variables, \pm 1 -valued, and generally, all bounded random variables. The subgaussian assumption is equivalent to the moment growth condition $\left(\mathbb{E}|X|^{p}\right)^{1 / p}=O(\sqrt{p})$ as $p \rightarrow \infty$.

Theorem 2.3 (Limiting distribution of extreme singular values, see [25]). Let $A=A_{N, n}$ be an $N \times n$ random matrix whose entries are independent and identically distributed subgaussian random variables with zero mean and unit variance. Suppose that the dimensions $N$ and $n$ grow to infinity while the aspect ratio $n / N$ stays uniformly bounded by some number $y \in(0,1)$. Then the normalized extreme singular values

$$
\frac{s_{\min }(A)^{2}-(\sqrt{N}-\sqrt{n})^{2}}{(\sqrt{N}-\sqrt{n})(1 / \sqrt{n}-1 / \sqrt{N})^{1 / 3}} \quad \text { and } \quad \frac{s_{\max }(A)^{2}-(\sqrt{N}+\sqrt{n})^{2}}{(\sqrt{N}+\sqrt{n})(1 / \sqrt{n}+1 / \sqrt{N})^{1 / 3}}
$$

converge in distribution to the Tracy-Widom law (2.2).

Non-asymptotic behavior of extreme singular values It is not entirely clear to what extent the limiting behavior of the extreme singular values such as asymptotics (2.1) manifests itself in fixed dimensions. Given the geometric meaning of the extreme singular values, our interest generally lies in establishing correct upper bounds on $s_{\max }(A)$ and lower bounds on $s_{\min }(A)$. We start with a folklore observation which yields the correct bound $s_{\max }(A) \lesssim \sqrt{N}+\sqrt{n}$ up to an absolute constant factor. The proof is a basic instance of an $\varepsilon$-net argument, a technique proved to be very useful in geometric functional analysis.

Proposition 2.4 (Largest singular value of subgaussian matrices: rough bound). Let $A$ be an $N \times n$ random matrix whose entries are independent mean zero subgaussian random variables whose subgaussian moments are bounded by 1 . Then

$$
\mathbb{P}\left(s_{\max }(A)>C(\sqrt{N}+\sqrt{n})+t\right) \leq 2 e^{-c t^{2}}, \quad t \geq 0 .
$$

Here and elsewhere in this paper, $C, C_{1}, c, c_{1}$ denote positive absolute constants. 
Proof (sketch). We will sketch the proof for $N=n$; the general case is similar. The expression $s_{\max }(A)=\max _{x, y \in S^{n-1}}\langle A x, y\rangle$ motivates us to first control the random variables $\langle A x, y\rangle$ individually for each pair of vectors $x, y$ on the unit Euclidean sphere $S^{n-1}$, and afterwards take the union bound over all such pairs. For fixed $x, y \in S^{n-1}$ the expression $\langle A x, y\rangle=\sum_{i, j} a_{i j} x_{j} y_{i}$ is a sum of independent random variables, where $a_{i j}$ denote the independent entries of $A$. If $a_{i j}$ were standard normal random variables, the rotation invariance of the Gaussian distribution would imply that $\langle A x, y\rangle$ is again a standard normal random variable. This property generalizes to subgaussian random variables. Indeed, using moment generating functions one can show that a normalized sum of mean zero subgaussian random variables is again a subgaussian random variable, although the subgaussian moment may increase by an absolute constant factor. Thus

$$
\mathbb{P}(\langle A x, y\rangle>s) \leq 2 e^{-c s^{2}}, \quad s \geq 0 .
$$

Obviously, we cannot finish the argument by taking the union bound over infinite (even uncountable) number of pairs $x, y$ on the sphere $S^{n-1}$. In order to reduce the number of such pairs, we discretize $S^{n-1}$ by considering its $\varepsilon$-net $\mathcal{N}_{\varepsilon}$ in the Euclidean norm, which is a subset of the sphere that approximates every point of the sphere up to error $\varepsilon$. An approximation argument yields

$$
s_{\max }(A)=\max _{x, y \in S^{n-1}}\langle A x, y\rangle \leq(1-\varepsilon)^{-2} \max _{x, y \in \mathcal{N}_{\varepsilon}}\langle A x, y\rangle \quad \text { for } \varepsilon \in(0,1) .
$$

To gain a control over the size of the net $\mathcal{N}_{\varepsilon}$, we construct it as a maximal $\varepsilon$ separated subset of $S^{n-1}$; then the balls with centers in $\mathcal{N}_{\varepsilon}$ and radii $\varepsilon / 2$ form a packing inside the centered ball of radius $1+\varepsilon / 2$. A volume comparison gives the useful bound on the cardinality of the net: $\left|\mathcal{N}_{\varepsilon}\right| \leq(1+2 / \varepsilon)^{n}$. Choosing for example $\varepsilon=1 / 2$, we are well prepared to take the union bound:

$$
\mathbb{P}\left(s_{\max }(A)>4 s\right) \leq \mathbb{P}\left(\max _{x, y \in \mathcal{N}_{\varepsilon}}\langle A x, y\rangle>s\right) \leq\left|\mathcal{N}_{\varepsilon}\right| \max _{x, y \in \mathcal{N}_{\varepsilon}} \mathbb{P}(\langle A x, y\rangle>s) \leq 5^{n} \cdot 2 e^{-c s^{2}} .
$$

We complete the proof by choosing $s=C \sqrt{n}+t$ with appropriate constant $C$.

By integration, one can easily deduce from Proposition 2.4 the correct expectation bound $\mathbb{E} s_{\max }(A) \leq C_{1}(\sqrt{N}+\sqrt{n})$. This latter bound actually holds under much weaker moment assumptions. Similarly to Theorem 2.1 the weakest possible fourth moment assumption suffices here. R. Latala [46] obtained the following general result for matrices with not identically distributed entries:

Theorem 2.5 (Largest singular value: fourth moment, non-iid entries [46]). Let $A$ be a random matrix whose entries $a_{i j}$ are independent mean zero random variables with finite fourth moment. Then

$$
\mathbb{E} s_{\max }(A) \leq C\left[\max _{i}\left(\sum_{j} \mathbb{E} a_{i j}^{2}\right)^{1 / 2}+\max _{j}\left(\sum_{i} \mathbb{E} a_{i j}^{2}\right)^{1 / 2}+\left(\sum_{i, j} \mathbb{E} a_{i j}^{4}\right)^{1 / 4}\right] .
$$

For random Gaussian matrices, a much sharper result than in Proposition 2.4 is due to Gordon [31, 32, 33]: 
Theorem 2.6 (Exteme singular values of Gaussian matrices, see [19]). Let A be an $N \times n$ matrix whose entries are independent standard normal random variables. Then

$$
\sqrt{N}-\sqrt{n} \leq \mathbb{E} s_{\min }(A) \leq \mathbb{E} s_{\max }(A) \leq \sqrt{N}+\sqrt{n} .
$$

This result is a consequence of the sharp comparison inequalities for Gaussian processes due to Slepian and Gordon, see [31, 32, 33, and [49, Section 3.3].

Tracy-Widom fluctuations One can deduce from Theorem [2.6 a deviation inequality for the extreme singular values. It follows formally by using the concentration of measure in the Gauss space. Since the $s_{\min }(A), s_{\max }(A)$ are 1-Lipschitz functions of $A$ considered as a vector in $\mathbb{R}^{N n}$, we have

$$
\mathbb{P}\left(\sqrt{N}-\sqrt{n}-t \leq s_{\min }(A) \leq s_{\max }(A) \leq \sqrt{N}+\sqrt{n}+t\right) \geq 1-2 e^{-t^{2} / 2}, \quad t \geq 0,
$$

see [19. For general random matrices with independent bounded entries, one can use Talagrand's concentration inequality for convex Lipschitz functions on the cube [76, 77. Namely, suppose the entries of $A$ are independent, have mean zero, and are uniformly bounded by 1 . Since $s_{\max }(A)$ is a convex function of $A$, Talagrand's concentration inequality implies

$$
\mathbb{P}\left(\left|s_{\max }(A)-\operatorname{Median}\left(s_{\max }(A)\right)\right| \geq t\right) \leq 2 e^{-t^{2} / 2} .
$$

Although the precise value of the median is unknown, integration of the previous inequality shows that $\left|\mathbb{E} s_{\max }(A)-\operatorname{Median}\left(s_{\max }(A)\right)\right| \leq C$. The same deviation inequality holds for symmetric random matrices.

Inequality (2.3) is optimal for large $t$ because $s_{\max }(A)$ is bounded below by the magnitude of every entry of $A$ which has the Gaussian tail. But for small deviations, say for $t<1$, inequality (2.3) is meaningless. Tracy-Widom law predicts a different tail behavior for small deviations $t$. It must follow the tail decay of the Tracy-Widom function $F_{1}$, which is not subgaussian [3], 39]:

$$
c \exp \left(-C \tau^{3 / 2}\right) \leq 1-F_{1}(\tau) \leq C \exp \left(-C^{\prime} \tau^{3 / 2}\right) \quad \tau \geq 0 .
$$

The concentration of this type for Hermitian complex and real Gaussian matrices (Gaussian Unitary Ensemble and Gaussian Orthogonal Ensemble) was proved by Ledoux [48 and Aubrun [3. Recently, Feldheim and Sodin [25] introduced a general approach, which allows to prove the asymptotic Tracy-Widom law and its non-asymptotic counterpart at the same time. Moreover, their method is applicable to random matrices with independent subgaussian entries both in symmetric and non-symmetric case. In particular, for an $N \times n$ random matrix $A$ with independent subgaussian entries they proved that

$$
p(\tau):=\mathbb{P}\left(s_{\max }(A) \geq \sqrt{N}+\sqrt{n}+\tau \sqrt{N}\right) \leq C \exp \left(-c n \tau^{3 / 2}\right) \quad \tau \geq 0 .
$$

Bounds (2.3) and (2.4) show that the tail behavior of the maximal singular value is essentially different for small and large deviations: $p(\tau)$ decays like $\exp \left(-c n \tau^{3 / 2}\right)$ 
for $\tau \leq c(n / N)^{2}$ and like $\exp \left(-c_{1} N \tau^{2}\right)$ for larger $\tau$. For square matrices the meaning of this phenomenon is especially clear. Large deviations of $s_{\max }(A)$ are produced by bursts of single entries: both $\mathbb{P}\left(s_{\max }(A) \geq \mathbb{E} s_{\max }(A)+t\right)$ and $\mathbb{P}\left(\left|a_{1,1}\right| \geq \mathbb{E} s_{\max }(A)+t\right)$ are of the same order $\exp \left(-c t^{2}\right)$ for $t \geq \mathbb{E} s_{\max }(A)$. In contrast, for small deviations (for smaller $t$ ) the situation becomes truly multidimensional, and Tracy-Widom type asymptotics appears.

The method of [25] also addresses the more difficult smallest singular value. For an $N \times n$ random matrix $A$ whose dimensions are not too close to each other Feldheim and Sodin [25] proved the Tracy-Widom law for the smallest singular value together with a non-asymptotic version of the bound $s_{\min }(A) \sim \sqrt{N}-\sqrt{n}$ :

$$
\mathbb{P}\left(s_{\min }(A) \leq \sqrt{N}-\sqrt{n}-\tau \sqrt{N} \cdot \frac{N}{N-n}\right) \leq \frac{C}{1-\sqrt{n / N}} \exp \left(-c^{\prime} n \tau^{3 / 2}\right) .
$$

\section{The smallest singular value}

Qualitative invertibility problem In this section we focus on the behavior of the smallest singular value of random $N \times n$ matrices with independent entries. The smallest singular value - the hard edge of the spectrum - is generally more difficult and less amenable to analysis by classical methods of random matrix theory than the largest singular value, the "soft edge". The difficulty especially manifests itself for square matrices $(N=n)$ or almost square matrices $(N-n=o(n))$. For example, we were guided so far by the asymptotic prediction $s_{\min }(A) \sim \sqrt{N}-\sqrt{n}$, which obviously becomes useless for square matrices.

A remarkable example is provided by $n \times n$ random Bernoulli matrices A, whose entries are independent \pm 1 valued symmetric random variables. Even the qualitative invertibility problem, which asks to estimate the probability that $A$ is invertible, is nontrivial in this situation. Komlós [44, 45] showed that $A$ is invertible asymptotically almost surely: $p_{n}:=\mathbb{P}\left(s_{\min }(A)=0\right) \rightarrow 0$ as $n \rightarrow \infty$. Later Kahn, Komlos and Szemeredi [43] proved that the singularity probability satisfies $p_{n} \leq c^{n}$ for some $c \in(0,1)$. The base $c$ was gradually improved in 78 , 81, with the latest record of $p_{n}=(1 / \sqrt{2}+o(1))^{n}$ obtained in [12]. It is conjectured that the dominant source of singularity of $A$ is the presence of two rows or two columns that are equal up to a sign, which would imply the best possible bound $p_{n}=(1 / 2+o(1))^{n}$.

Quantitative invertibility problem The previous problem is only concerned with whether the hard edge $s_{\min }(A)$ is zero or not. This says nothing about the quantitative invertibility problem of the typical size of $s_{\min }(A)$. The latter question has a long history. Von Neumann and his associates used random matrices as test inputs in algorithms for numerical solution of systems of linear equations. The accuracy of the matrix algorithms, and sometimes their running time as well, depends on the condition number $\kappa(A)=s_{\max }(A) / s_{\min }(A)$. Based on heuristic and experimental evidence, von Neumann and Goldstine predicted that

$$
s_{\min }(A) \sim n^{-1 / 2}, \quad s_{\max }(A) \sim n^{1 / 2} \quad \text { with high probability }
$$


which together yield $\kappa(A) \sim n$, see [92, Section 7.8]. In Section 2 we saw several results establishing the second part of (3.1), for the largest singular value.

Estimating the smallest singular value turned out to be more difficult. A more precise form of the prediction $s_{\min }(A) \sim n^{-1 / 2}$ was repeated by Smale 73 and proved by Edelman [20] and Szarek [79] for random Gaussian matrices A, those with i.i.d. standard normal entries. For such matrices, the explicit formula for the joint density of the eigenvalues $\lambda_{i}$ of $\frac{1}{n} A^{*} A$ is available:

$$
\operatorname{pdf}\left(\lambda_{1}, \ldots, \lambda_{n}\right)=C_{n} \prod_{1 \leq i<j \leq n}\left|\lambda_{i}-\lambda_{j}\right| \prod_{i=1}^{n} \lambda_{i}^{-1 / 2} \exp \left(-\sum_{i=1}^{n} \lambda_{i} / 2\right) .
$$

Integrating out all the eigenvalues except the smallest one, one can in principle compute its distribution. This approach leads to the following asymptotic result:

Theorem 3.1 (Smallest singular value of Gaussian matrices [20]). Let $A=A_{n}$ be an $n \times n$ random matrix whose entries are independent standard normal random variables. Then for every fixed $\varepsilon \geq 0$ one has

$$
\mathbb{P}\left(s_{\min }(A) \leq \varepsilon n^{-1 / 2}\right) \rightarrow 1-\exp \left(-\varepsilon-\varepsilon^{2} / 2\right) \quad \text { as } n \rightarrow \infty .
$$

The limiting probability behaves as $1-\exp \left(-\varepsilon-\varepsilon^{2} / 2\right) \sim \varepsilon$ for small $\varepsilon$. In fact, the following non-asymptotic bound holds for all $n$ :

$$
\mathbb{P}\left(s_{\min }(A) \leq \varepsilon n^{-1 / 2}\right) \leq \varepsilon, \quad \varepsilon \geq 0 .
$$

This follows from the analysis of Edelman [20; Sankar, Spielman and Teng 68] provided a different geometric proof of estimate (3.2) up to an absolute constant factor and extended it to non-centered Gaussian distributions.

Smallest singular values of general random matrices These methods do not work for general random matrices, especially those with discrete distributions, where rotation invariance and the joint density of eigenvalues are not available. The prediction that $s_{\min }(A) \sim n^{-1 / 2}$ has been open even for random Bernoulli matrices. Spielman and Teng conjectured in their ICM 2002 talk 75 that estimate (3.2) should hold for the random Bernoulli matrices up to an exponentially small term that accounts for their singularity probability:

$$
\mathbb{P}\left(s_{\min }(A) \leq \varepsilon n^{-1 / 2}\right) \leq \varepsilon+c^{n}, \quad \varepsilon \geq 0
$$

where $c \in(0,1)$ is an absolute constant. The first polynomial bound on $s_{\min }(A)$ for general random matrices was obtained in $[63$. Later Spielman-Teng's conjecture was proved in 65] up to a constant factor, and for general random matrices:

Theorem 3.2 (Smallest singular value of square random matrices 65]). Let $A$ be an $n \times n$ random matrix whose entries are independent and identically distributed subgaussian random variables with zero mean and unit variance. Then

$$
\mathbb{P}\left(s_{\min }(A) \leq \varepsilon n^{-1 / 2}\right) \leq C \varepsilon+c^{n}, \quad \varepsilon \geq 0
$$

where $C>0$ and $c \in(0,1)$ depend only on the subgaussian moment of the entries. 
This result addresses both qualitative and quantitative aspects of the invertibility problem. Setting $\varepsilon=0$ we see that $A$ is invertible with probability at least $1-c^{n}$. This generaizes the result of Kahn, Komlos and Szemeredi 43 from Bernoulli to all subgaussian matrices. On the other hand, quantitatively, Theorem 3.2 states that $s_{\min }(A) \gtrsim n^{-1 / 2}$ with high probability for general random matrices. A corresponding non-asymptotic upper bound $s_{\min }(A) \lesssim n^{-1 / 2}$ also holds [66], so we have $s_{\min }(A) \sim n^{-1 / 2}$ as in von Neumann-Goldstine's prediction. Both these bounds, upper and lower, hold with high probability under the weaker fourth moment assumption on the entries [65, 66].

This theory was extended to rectangular random matrices of arbitrary dimensions $N \times n$ in 67. As we know from Section 2, one expects that $s_{\min }(A) \sim$ $\sqrt{N}-\sqrt{n}$. But this would be incorrect for square matrices. To reconcile rectangular and square matrices we make the following correction of our prediction:

$$
s_{\min }(A) \sim \sqrt{N}-\sqrt{n-1} \text { with high probability. }
$$

For square matrices one would have the correct estimate $s_{\min }(A) \sim \sqrt{n}-\sqrt{n-1} \sim$ $n^{-1 / 2}$. The following result extends Theorem 3.2 to rectangular matrices:

Theorem 3.3 (Smallest singular value of rectangular random matrices [65]). Let A be an $n \times n$ random matrix whose entries are independent and identically distributed subgaussian random variables with zero mean and unit variance. Then

$$
\mathbb{P}\left(s_{\min }(A) \leq \varepsilon(\sqrt{N}-\sqrt{n-1})\right) \leq(C \varepsilon)^{N-n+1}+c^{N}, \quad \varepsilon \geq 0
$$

where $C>0$ and $c \in(0,1)$ depend only on the subgaussian moment of the entries.

This result has been known for a long time for tall matrices, whose the aspect ratio $\lambda=n / N$ is bounded by a sufficiently small constant, see [10. The optimal bound $s_{\min }(A) \geq c \sqrt{N}$ can be proved in this case using an $\varepsilon$-net argument similar to Proposition 2.4. This was extended in [53] to $s_{\min }(A) \geq c_{\lambda} \sqrt{N}$ for all aspect ratios $\lambda<1-c / \log n$. The dependence of $c_{\lambda}$ on the aspect ratio $\lambda$ was improved in 2 for Bernoulli matrices and in 62 for general subgaussian matrices. FeldheimSodin's Theorem 2.3 gives precise Tracy-Widom fluctuations of $s_{\min }(A)$ for tall matrices, but becomes useless for almost square matrices (say for $N<n+n^{1 / 3}$ ). Theorem 3.3 is an an optimal result (up to absolute constants) which covers matrices with all aspect ratios from tall to square. Non-asymptotic estimate (3.3) was extended to matrices whose entries have finite $(4+\varepsilon)$-th moment in 93 .

Universality of the smallest singular values The limiting distribution of $s_{\text {min }}(A)$ turns out to be universal as dimension $n \rightarrow \infty$. We already saw a similar universality phenomenon in Theorem 2.3 for genuinely rectangular matrices. For square matrices, the corresponding result was proved by Tao and $\mathrm{Vu}$ [87]:

Theorem 3.4 (Smallest singular value of square matrices: universality [87]). Let A be an $n \times n$ random matrix whose entries are independent and identically distributed random variables with zero mean, unit variance, and finite $K$-th moment where $K$ 
is a sufficiently large absolute constant. Let $G$ be an $n \times n$ random matrix whose entries are independent standard normal random variables. Then

$\mathbb{P}\left(\sqrt{n} s_{\min }(G) \leq t-n^{-c}\right)-n^{c} \leq \mathbb{P}\left(\sqrt{n} s_{\min }(A) \leq t\right) \leq \mathbb{P}\left(\sqrt{n} s_{\min }(G) \leq t+n^{-c}\right)+n^{c}$

where $c>0$ depends only on the $K$-th moment of the entries.

On a methodological level, this result may be compared in classical probability theory to Berry-Esseen theorem (1.1) which establishes polynomial deviations from the limiting distribution, while Theorems 3.2 and 3.3 bear a similarity with large deviation results like (1.2) which give exponentially small tail probabilities.

Sparsity and invertibility: a geometric proof of Theorem 3.2 We will now sketch the proof of Theorem 3.2 given in [65. This argument is mostly based on geometric ideas, and it may be useful beyond spectral analysis of random matrices.

Looking at $s_{\min }(A)=\min _{x \in S^{n-1}}\|A x\|_{2}$ we see that our goal is to bound below $\|A x\|_{2}$ uniformly for all unit vectors $x$. We will do this separately for sparse vectors and for spread vectors with two very different arguments. Choosing a small absolute constant $c_{0}>0$, we first consider the class of sparse vectors

$$
\text { Sparse }:=\left\{x \in S^{n-1}:|\operatorname{supp}(x)| \leq c_{0} n\right\}
$$

Establishing invertibility of $A$ on this class is relatively easy. Indeed, when we look at $\|A x\|_{2}$ for sparse vectors $x$ of fixed $\operatorname{support} \operatorname{supp}(x)=I$ of size $|I|=c_{0} n$, we are effectively dealing with the $n \times c_{0} n$ submatrix $A_{I}$ that consists of the columns of $A$ indexed by $I$. The matrix $A_{I}$ is tall, so as we said below Theorem 3.3. its smallest singular value can be estimated using the standard $\varepsilon$-net argument. This gives $s_{\min }\left(A_{I}\right) \geq c n^{1 / 2}$ with probability at least $1-2 e^{-n}$. This allows us to further take the union bound over $\left(\begin{array}{c}n \\ c_{0} n\end{array}\right) \leq e^{n / 2}$ choices of support $I$, and conclude that with probability at least $1-2 e^{-n / 2}$ we have invertibility on all sparse vectors:

$$
\min _{x \in \text { Sparse }}\|A x\|_{2}=\min _{|I| \leq c_{0} n} s_{\min }\left(A_{I}\right) \geq c n^{1 / 2} .
$$

We thus obtained a much stronger bound than we need, $n^{1 / 2}$ instead of $n^{-1 / 2}$.

Establishing invertibility of $A$ on non-sparse vectors is more difficult because there are too many of them. For example, there are exponentially many vectors on $S^{n-1}$ whose coordinates all equal $\pm n^{-1 / 2}$ and which have at least a constant distance from each other. This gives us no hope to control such vectors using $\varepsilon$-nets, as any nontrivial net must have cardinality at least $2^{n}$. So let us now focus on this most difficult class of extremely non-sparse vectors

$$
\text { Spread }:=\left\{x \in S^{n-1}:\left|x_{i}\right| \geq c_{1} n^{-1 / 2} \text { for all } i\right\} .
$$

Once we prove invertibility of $A$ on these spread vectors, the argument can be completed for all vectors in $S^{n-1}$ by an approximation argument. Loosely speaking, if $x$ is close to Sparse we can treat $x$ as sparse, otherwise $x$ must have at least $c n$ coordinates of magnitude $\left|x_{i}\right|=O\left(n^{-1 / 2}\right)$, which allows us to treat $x$ as spread. 
An obvious advantage of spread vectors is that we know the magnitude of all their coefficients. This motivates the following geometric invertibility argument. If $A$ performs extremely poor so that $s_{\min }(A)=0$, then one of the columns $X_{k}$ of $A$ lies in the span $H_{k}=\operatorname{span}\left(X_{i}\right)_{i \neq k}$ of the others. This simple observation can be transformed into a quantitative argument. Suppose $x=\left(x_{1}, \ldots, x_{n}\right) \in \mathbb{R}^{n}$ is a spread vector. Then, for every $k=1, \ldots, n$, we have

$$
\begin{aligned}
\|A x\|_{2} & \geq \operatorname{dist}\left(A x, H_{k}\right)=\operatorname{dist}\left(\sum_{i=1}^{n} x_{i} X_{i}, H_{k}\right)=\operatorname{dist}\left(x_{k} X_{k}, H_{k}\right) \\
& =\left|x_{k}\right| \cdot \operatorname{dist}\left(X_{k}, H_{k}\right) \geq c_{1} n^{-1 / 2} \operatorname{dist}\left(X_{k}, H_{k}\right) .
\end{aligned}
$$

Since the right hand side does not depend on $x$, we have proved that

$$
\min _{x \in \text { Spread }}\|A x\|_{2} \geq c_{1} n^{-1 / 2} \operatorname{dist}\left(X_{n}, H_{n}\right) .
$$

This reduces our task to the geometric problem of independent interest - estimate the distance between a random vector and an independent random hyperplane. The expectation estimate $1 \leq \mathbb{E} \operatorname{dist}\left(X_{n}, H_{n}\right)^{2}=O(1)$ follows easily by independence and moment assumptions. But we need a lower bound with high probability, which is far from trivial. This will make a separate story connected to the Littlewood-Offord theory of small ball probabilities, which we discuss in Section 4. In particular we will prove in Corollary 4.4 the optimal estimate

$$
\mathbb{P}\left(\operatorname{dist}\left(X_{n}, H_{n}\right) \leq \varepsilon\right) \leq C \varepsilon+c^{n}, \quad \varepsilon \geq 0,
$$

which is simple for the Gaussian distribution (by rotation invariance) and difficult to prove e.g. for the Bernoulli distribution. Together with (3.6) this means that we proved invertibility on all spread vectors:

$$
\mathbb{P}\left(\min _{x \in \text { Spread }}\|A x\|_{2} \leq \varepsilon n^{-1 / 2}\right) \leq C \varepsilon+c^{n}, \quad \varepsilon \geq 0 .
$$

This is exactly the type of probability bound claimed in Theorem 3.2 As we said, we can finish the proof by combining with the (much better) invertibility on sparse vectors in (3.4), and by an approximation argument.

\section{Littlewood-Offord theory}

Small ball probabilities and additive structure We encountered the following geometric problem in the previous section: estimate the distance between a random vector $X$ with independent coordinates and an independent random hyperplane $H$ in $\mathbb{R}^{n}$. We need a lower bound on this distance with high probability. Let us condition on the hyperplane $H$ and let $a \in \mathbb{R}^{n}$ denote its unit normal vector. Writing in coordinates $a=\left(a_{1}, \ldots, a_{n}\right)$ and $X=\left(\xi_{1}, \ldots, \xi_{n}\right)$, we see that

$$
\operatorname{dist}(X, H)=\langle a, X\rangle=\left|\sum_{i=1}^{n} a_{i} \xi_{i}\right|
$$


We need to understand the distribution of sums of independent random variables

$$
S=\sum_{i=1}^{n} a_{i} \xi_{i}, \quad\|a\|_{2}=1
$$

where $a=\left(a_{1}, \ldots, a_{n}\right) \in \mathbb{R}^{n}$ is a given coefficient vector, and $\xi_{1}, \ldots, \xi_{n}$ are independent identically distributed random variables with zero mean and unit variance.

Sums of independent random variables is a classical theme in probability theory. The well-developed area of large deviation inequalities like (1.2) demonstrates that $S$ nicely concentrates around its mean. But our problem is opposite as we need to show that $S$ is not too concentrated around its mean 0 , and perhaps more generally around any real number. Several results in probability theory starting from the works of Lévy [50, Kolmogorov 42 and Esséen 24 were concerned with the spread of sums of independent random variables, which is quantified as follows:

Definition 4.1. The Lévy concentration function of a random variable $S$ is

$$
\mathcal{L}(S, \varepsilon)=\sup _{v \in \mathbb{R}} \mathbb{P}(|S-v| \leq \varepsilon), \quad \varepsilon \geq 0
$$

Lévy concentration function measures the small ball probability [51, the likelihood that $S$ enters a small interval. For continuous distributions one can show that $\mathcal{L}(S, \varepsilon) \lesssim \varepsilon$ for all $\varepsilon \geq 0$. For discrete distributions this may be false. Instead, a new phenomenon arises for discrete distributions which is unseen in large deviation theory: Lévy concentration function depends on the additive structure of the coefficient vector $a$. This is best illustrated on the example where $\xi_{i}$ are independent Bernoulli random variables ( \pm 1 valued and symmetric). For sparse vectors like $a=2^{-1 / 2}(1,1,0, \ldots, 0)$, Lévy concentration function can be large: $\mathcal{L}(S, 0)=1 / 2$. For spread vectors, Berry-Esseen's theorem (1.1) yields a better bound:

$$
\text { For } a^{\prime}=n^{-1 / 2}(1,1, \ldots, 1), \quad \mathcal{L}(S, \varepsilon) \leq C\left(\varepsilon+n^{-1 / 2}\right) .
$$

The threshold $n^{-1 / 2}$ comes from many cancelations in the sums $\sum \pm 1$ which occur because all coefficients $a_{i}$ are equal. For less structured $a$, fewer cancelations occur:

$$
\text { For } a^{\prime \prime}=n^{-1 / 2}\left(1+\frac{1}{n}, 1+\frac{2}{n}, \ldots, 1+\frac{n}{n}\right), \quad \mathcal{L}(S, 0) \sim n^{-3 / 2} .
$$

Studying the influence of additive structure of the coefficient vector $a$ on the spread of $S=\sum a_{i} \xi_{i}$ became known as the Littlewood-Offord problem. It was initially developed by Littlewood and Offord [52, Erdös and Moser 21, 22, Sárkozy and Szemerédi 69, Halasz [40, Frankl and Füredi [26]. For example, if all $\left|a_{i}\right| \geq 1$ then $\mathcal{L}(S, 1) \leq C n^{-1 / 2}$ [52, 21], which agrees with (4.2). Similarly, a general fact behind (4.3) is that if $\left|a_{i}-a_{j}\right| \geq 1$ for all $i \neq j$ then $\mathcal{L}(S, 1) \leq C n^{-3 / 2}$ [22, 69, 40].

New results on Lévy concentration function Problems of invertibility of random matrices motivated a recent revisiting of the Littlewood-Offord problem by Tao and $\mathrm{Vu}$ [83, 84, 86, 88, the authors [65, 67], Friedland and Sodin [27]. 
Additive structure of the coefficient vector $a$ is related to the shortest arithmetic progression into which it embeds. This length is conveniently expressed as the least common denominator $\operatorname{lcd}(a)$ defined as the smallest $\theta>0$ such that $\theta a \in$ $\mathbb{Z}^{n} \backslash 0$. Examples suggest that Lévy concentration function should be inversely proportional to the least common denominator: $\operatorname{lcd}\left(a^{\prime}\right)=n^{1 / 2} \sim 1 / \mathcal{L}(S, 0)$ in (4.2) and $\operatorname{lcd}\left(a^{\prime \prime}\right)=n^{3 / 2} \sim 1 / \mathcal{L}(S, 0)$ in (4.3). This is not a coincidence. But to state a general result, we will need to consider a more stable version of the least common denominator. Given an accuracy level $\alpha>0$, we define the essential least common denominator

$$
\operatorname{lcd}_{\alpha}(a):=\inf \left\{\theta>0: \operatorname{dist}\left(\theta a, \mathbb{Z}^{n}\right) \leq \min \left(\frac{1}{10}\|\theta a\|_{2}, \alpha\right)\right\} .
$$

The requirement $\operatorname{dist}\left(\theta a, \mathbb{Z}^{n}\right) \leq \frac{1}{10}\|\theta a\|_{2}$ ensures approximation of $\theta a$ by non-trivial integer points, those in a non-trivial cone in the direction of $a$. The constant $\frac{1}{10}$ is arbitrary and it can be replaced by any other constant in $(0,1)$. One typically uses this concept for accuracy levels $\alpha=c \sqrt{n}$ with a small constant $c$ such as $c=\frac{1}{10}$. The inequality $\operatorname{dist}\left(\theta a, \mathbb{Z}^{n}\right) \leq \alpha$ yields that most of the coordinates of $\theta a$ are within a small constant distance from integers. For such $\alpha$, in examples (4.2) and (4.3) one has as before $\operatorname{lcd}_{\alpha}\left(a^{\prime}\right) \sim n^{1 / 2}$ and $\operatorname{lcd}_{\alpha}\left(a^{\prime \prime}\right) \sim n^{3 / 2}$. Here we state and sketch a proof of a general Littlewood-Offord type result from [67.

Theorem 4.2 (Lévy concentration function via additive structure). Let $\xi_{1}, \ldots, \xi_{n}$ be independent identically distributed mean zero random variables, which are well spread: $p:=\mathcal{L}\left(\xi_{k}, 1\right)<1$. Then, for every coefficient vector $a=\left(a_{1}, \ldots, a_{n}\right) \in$ $S^{n-1}$ and every accuracy level $\alpha>0$, the sum $S=\sum_{i=1}^{n} a_{i} \xi_{i}$ satisfies

$$
\mathcal{L}(S, \varepsilon) \leq C \varepsilon+C / \operatorname{lcd}_{\alpha}(a)+C e^{-c \alpha^{2}}, \quad \varepsilon \geq 0,
$$

where $C, c>0$ depend only on the spread $p$.

Proof. A classical Esseen's concentration inequality [24] bounds the Lévy concentration function of an arbitrary random variable $Z$ by the $L_{1}$ norm of its characteristic function $\phi_{Z}(\theta)=\mathbb{E} \exp (i \theta Z)$ as follows:

$$
\mathcal{L}(Z, 1) \leq C \int_{-1}^{1}\left|\phi_{Z}(\theta)\right| d \theta
$$

One can prove this inequality using Fourier inversion formula, see [80, Section 7.3].

We will show how to prove Theorem 4.2 for Bernoulli random variables $\xi_{i}$; the general case requires an additional argument. Without loss of generality we can assume that $\operatorname{lcd}_{\alpha}(a) \geq \frac{1}{\pi \varepsilon}$. Applying (4.5) for $Z=S / \varepsilon$, we obtain by independence that

$$
\mathcal{L}(S, \varepsilon) \leq C \int_{-1}^{1}\left|\phi_{S}(\theta / \varepsilon)\right| d \theta=C \int_{-1}^{1} \prod_{j=1}^{n}\left|\phi_{j}(\theta / \varepsilon)\right| d \theta,
$$

where $\phi_{j}(t)=\mathbb{E} \exp \left(i a_{j} \xi_{j} t\right)=\cos \left(a_{j} t\right)$. The inequality $|x| \leq \exp \left(-\frac{1}{2}\left(1-x^{2}\right)\right)$ which is valid for all $x \in \mathbb{R}$ implies that

$$
\left|\phi_{j}(t)\right| \leq \exp \left(-\frac{1}{2} \sin ^{2}\left(a_{j} t\right)\right) \leq \exp \left(-\frac{1}{2} \operatorname{dist}\left(\frac{a_{j} t}{\pi}, \mathbb{Z}\right)^{2}\right) .
$$


Therefore

$$
\mathcal{L}(S, \varepsilon) \leq C \int_{-1}^{1} \exp \left(-\frac{1}{2} \sum_{j=1}^{n} \operatorname{dist}\left(\frac{a_{j} \theta}{\pi \varepsilon}, \mathbb{Z}\right)^{2}\right) d \theta=C \int_{-1}^{1} \exp \left(-\frac{1}{2} f^{2}(\theta)\right) d \theta
$$

where $f(\theta)=\operatorname{dist}\left(\frac{\theta}{\pi \varepsilon} a, \mathbb{Z}^{n}\right)$. Since $\operatorname{lcd}_{\alpha}(a) \geq \frac{1}{\pi \varepsilon}$, the definition of the essential least common denominator implies that for every $\theta \in[-1,1]$ we have $f(\theta) \geq$ $\min \left(\frac{\theta}{10 \pi \varepsilon}\|a\|_{2}, \alpha\right)$. Since by assumption $\|a\|_{2}=1$, it follows that

$$
\exp \left(-\frac{1}{2} f^{2}(\theta)\right) \leq \exp \left(-\frac{1}{2}\left(\frac{\theta}{10 \pi \varepsilon}\right)^{2}\right)+\exp \left(-\alpha^{2} / 2\right) .
$$

Substituting this into (4.6) yields $\mathcal{L}(S, \varepsilon) \leq C_{1}\left(\varepsilon+2 \exp \left(-\alpha^{2} / 2\right)\right)$ as required.

Theorem 4.2 justifies our empirical observation that Lévy concentration function is proportional to the amount of structure in the coefficient vector, which is measured by the (reciprocal of) its essential least common denominator. As we said, this result is typically used for accuracy level $\alpha=c \sqrt{n}$ with some small positive constant $c$. In this case, the term $C e^{-c \alpha^{2}}$ in (4.4) is exponentially small in $n$ (thus negligible in applications), and the term $C \varepsilon$ is optimal for continuous distributions.

Theorem 4.2 performs best for totally unstructured coefficient vectors $a$, those with exponentially large $\operatorname{lcd}_{\alpha}(a)$. Heuristically, this should be the case for random vectors, as randomness should destroy any structure. While this is not true for general vectors with independent coordinates (e.g. for equal coordinates with random signs), it is true for normals of random hyperplanes:

Theorem 4.3 (Random vectors are unstructured [65). Let $X_{i}$ be random vectors in $\mathbb{R}^{n}$ whose coordinates are independent and identically distributed subgaussian random variables with zero mean and unit variance. Let $a \in \mathbb{R}^{n}$ denote a unit normal vector of $H=\operatorname{span}\left(X_{1}, \ldots, X_{n-1}\right)$. Then, with probability at least $1-e^{-c n}$,

$$
\operatorname{lcd}_{\alpha}(a) \geq e^{c n} \quad \text { for } \alpha=c \sqrt{n},
$$

where $c>0$ depends only on the subgaussian moment.

Therefore for random normals $a$, Theorem 4.2 yealds with high probability a very strong bound on Lévy concentration function:

$$
\mathcal{L}(S, \varepsilon) \leq C \varepsilon+c^{n}, \quad \varepsilon \geq 0 .
$$

This brings us back to the distance problem considered in the beginning of this section, which motivated our study of Lévy concentration function:

Corollary 4.4 (Distance between random vectors and hyperplanes [65]). Let $X_{i}$ be random vectors as in Theorem 4.3, and $H_{n}=\operatorname{span}\left(X_{1}, \ldots, X_{n-1}\right)$. Then

$$
\mathbb{P}\left(\operatorname{dist}\left(X_{n}, H_{n}\right) \leq \varepsilon\right) \leq C \varepsilon+c^{n}, \quad \varepsilon \geq 0,
$$

where $C, c>0$ depend only on the subgaussian moment. 
Proof. As was noticed in (4.1), we can write $\operatorname{dist}\left(X_{n}, H_{n}\right)$ as a sum of independent random variables, and then bound it using (4.7).

Corollary 4.4 offers us exactly the missing piece (3.7) in our proof of the invertibility Theorem 3.2. This completes our analysis of invertibility of square matrices.

Remark. These methods generalize to rectangular matrices [67, 93]. For example, Corollary 4.4 can be extended to compute the distance between random vectors and subspaces of arbitrary dimension [67]: for $H_{n}=\operatorname{span}\left(X_{1}, \ldots, X_{n-d}\right)$ we have $\left(\mathbb{E} \operatorname{dist}\left(X_{n}, H_{n}\right)^{2}\right)^{1 / 2}=\sqrt{d}$ and

$$
\mathbb{P}\left(\operatorname{dist}\left(X_{n}, H_{n}\right) \leq \varepsilon \sqrt{d}\right) \leq(C \varepsilon)^{d}+c^{n}, \quad \varepsilon \geq 0 .
$$

\section{Applications}

The applications of non-asymptotic theory of random matrices are numerous, and we cannot cover all of them in this note. Instead we concentrate on three different results pertaining to the classical random matrix theory (Circular Law), signal processing (compressed sensing), and geometric functional analysis and theoretical computer science (short Khinchin's inequality and Kashin's subspaces).

Circular law Asymptotic theory of random matrices provides an important source of heuristics for non-asymptotic results. We have seen an illustration of this in the analysis of the extreme singular values. This interaction between the asymptotic and non-asymptotic theories goes the other way as well, as good nonasymptotic bounds are sometimes crucial in proving the limit laws. One remarkable example of this is the circular law which we will discuss now.

Consider a family of $n \times n$ matrices $A$ whose entries are independent copies of a random variable $X$ with mean zero and unit variance. Let $\mu_{n}$ be the empirical measure of the eigenvalues of the matrix $B_{n}=\frac{1}{\sqrt{n}} A_{n}$, i.e. the Borel probability measure on $\mathbb{C}$ such that $\mu_{n}(E)$ is the fraction of the eigenvalues of $B_{n}$ contained in $E$. A long-standing conjecture in random matrix theory, which is called the circular law, suggested that the measures $\mu_{n}$ converge to the normalized Lebesgue measure on the unit disc. The convergence here can be understood in the same sense as in the Wigner's semicircle law. The circular law was originally proved by Mehta [56] for random matrices with standard normal entries. The argument used the explicit formula for joint density of the eigenvalues, so it could not be extended to other classes of random matrices. While the formulation of Wigner's semicircle law and the circular law look similar, the methods used to prove the former are not applicable to the latter. The reason is that the spectrum of a general matrix, unlike that of a Hermitian matrix, is unstable: a small change of the entries may cause a significant change of the spectrum (see [6]). Girko [30] introduced a new approach to the circular law based on considering the real part of the Stieltjes transform of measures $\mu_{n}$. For $z=x+i y$ the real Stieltjes transform is defined by 
the formula

$$
S_{n r}(z)=\operatorname{Re}\left(\frac{1}{n} \operatorname{Tr}\left(B_{n}-z I_{n}\right)^{-1}\right)=-\frac{\partial}{\partial x}\left(\frac{1}{n} \log \left|\operatorname{det}\left(B_{n}-z I\right)\right|\right) .
$$

Since $\left|\operatorname{det}\left(B_{n}-z I\right)\right|^{2}=\operatorname{det}\left(B_{n}-z I\right)\left(B_{n}-z I\right)^{*}$, this is the same as

$$
S_{n r}(z)=-\frac{1}{2} \frac{\partial}{\partial x}\left(\frac{1}{n} \log \left|\operatorname{det}\left(B_{n}-z I\right)\left(B_{n}-z I\right)^{*}\right|\right)=-\frac{1}{2} \frac{\partial}{\partial x}\left(\frac{1}{n} \sum_{j=1}^{n} \log s_{j}^{(n)}(z)\right),
$$

where $s_{1}^{(n)}(z) \geq \ldots \geq s_{n}^{(n)}(z) \geq 0$ are the eigenvalues of the Hermitian matrix $\left(B_{n}-z I\right)\left(B_{n}-z I\right)^{*}$, or in other words, the squares of the singular values of the matrix $V_{n}=B_{n}-z I$. Girko's argument reduces the proof of the circular law to the convergence of real Stieltjes transforms, and thus to the behavior of the sum above. The logarithmic function is unbounded at 0 and $\infty$. To control the behavior near $\infty$, one has to use the bound for the largest singular value of $V_{n}$, which is relatively easy. The analysis of the behavior near 0 requires bounds on the smallest singular value of $V_{n}$, and is therefore more difficult.

Girko's approach was implemented by Bai 4, who proved the circular law for random matrices whose entries have bounded sixth moment and bounded density. The bounded density condition was sufficient to take care of the smallest singular value problem. This result was the first manifestation of the universality of the circular law. Still, it did not cover some important classes of random matrices, in particular random Bernoulli matrices. The recent results on the smallest singular value led to a significant progress on establishing the universality of the circular law. A crucial step was done by Götze and Tikhomirov [34 who extended the circular law to all subgaussian matrices using [63. In fact, the results of 34 actually extended it to all random entries with bounded fourth moment. This was further extended to random variables having bounded moment $2+\varepsilon$ in 35,82 . Finally, in 85 Tao and $\mathrm{Vu}$ proved the Circular Law in full generality, with no assumptions besides the unit variance. Their approach was based on the smallest singular value bound from 82 and a novel replacement principle which allowed them to treat the other singular values.

Compressed Sensing Non-asymptotic random matrix theory provides a right context for the analysis of random measurements in the newly developed area of compressed sensing, see the ICM 2006 talk of Candes [14. Compressed sensing is an area of information theory and signal processing which studies efficient techniques to reconstruct a signal from a small number of measurements by utilizing the prior knowledge that the signal is sparse [18].

Mathematically, one seeks to reconstruct an unknown signal $x \in \mathbb{R}^{n}$ from some $m$ linear measurements viewed as a vector $A x \in \mathbb{R}^{m}$, where $A$ is some known $m \times n$ matrix called the measurement matrix. In the interesting case $m<n$, the problem is underdetermined and we are interested in the sparsest solution:

$$
\text { minimize }\left\|x^{*}\right\|_{0} \text { subject to } A x^{*}=A x \text {, }
$$


where $\|x\|_{0}=|\operatorname{supp}(x)|$. This optimization problem is highly non-convex and computationally intractable. So one considers the following convex relaxation of (5.1), which can be efficiently solved by convex programming methods:

$$
\text { minimize }\left\|x^{*}\right\|_{1} \text { subject to } A x^{*}=A x \text {, }
$$

where $\|x\|_{1}=\sum_{i=1}^{n}\left|x_{i}\right|$ denotes the $\ell_{1}$ norm.

One would then need to find conditions when problems (5.1) and (5.2) are equivalent. Candes and Tao [16] showed that this occurs when the measurement matrix $A$ is a restricted isometry. For an integer $s \leq n$, the restricted isometry constant $\delta_{s}(A)$ is the smallest number $\delta \geq 0$ which satisfies

$$
(1-\delta)\|x\|_{2}^{2} \leq\|A x\|_{2}^{2} \leq(1+\delta)\|x\|_{2}^{2} \quad \text { for all } x \in \mathbb{R}^{n},|\operatorname{supp}(x)| \leq s .
$$

Geometrically, the restricted isometry property guarantees that the geometry of $s$-sparse vectors $x$ is well preserved by the measurement matrix $A$. In turns out that in this situation one can reconstruct $x$ from $A x$ by the convex program (5.2):

Theorem 5.1 (Sparse reconstruction using convex programming 16]). Assume $\delta_{2 s} \leq c$. Then the solution of (5.2) equals $x$ whenever $|\operatorname{supp}(x)| \leq s$.

A proof with $c=\sqrt{2}-1$ is given in [15]; the current record is $c=0.472$ [13].

Restricted isometry property can be interpreted in terms of the extreme singular values of submatrices of $A$. Indeed, (5.3) equivalently states that the inequality

$$
\sqrt{1-\delta} \leq s_{\min }\left(A_{I}\right) \leq s_{\max }\left(A_{I}\right) \leq \sqrt{1+\delta}
$$

holds for all $m \times s$ submatrices $A_{I}$, those formed by the columns of $A$ indexed by sets $I$ of size $s$. In light of Sections 2 and 3, it is not surprising that the best known restricted isometry matrices are random matrices. It is actually an open problem to construct deterministic restricted isometry matrices as in Theorem 5.2 below.

The following three types of random matrices are extensively used as measurement matrices in compressed sensing: Gaussian, Bernoulli, and Fourier. Here we summarize their restricted isometry properties, which have the common remarkable feature: the required number of measurements $m$ is roughly proportional to the sparsity level $s$ rather than the (possibly much larger) dimension $n$.

Theorem 5.2 (Random matrices are restricted isometries). Let $m, n, s$ be positive integers, $\varepsilon, \delta \in(0,1)$, and let $A$ be an $m \times n$ measurement matrix.

1. Suppose the entries of $A$ are independent and identically distributed subgaussian random variables with zero mean and unit variance. Assume that

$$
m \geq C s \log (2 n / s)
$$

where $C$ depends only on $\varepsilon, \delta$, and the subgaussian moment. Then with probability at least $1-\varepsilon$, the matrix $\bar{A}=\frac{1}{\sqrt{m}} A$ is a restricted isometry with $\delta_{s}(\bar{A}) \leq \delta$.

2. Let $A$ be a random Fourier matrix obtained from the $n \times n$ discrete Fourier transform matrix by choosing $m$ rows independently and uniformly. Assume that

$$
m \geq C s \log ^{4}(2 n) .
$$


where $C$ depends only on $\varepsilon$ and $\delta$. Then with probability at least $1-\varepsilon$, the matrix $\bar{A}=\frac{1}{\sqrt{n}} A$ is a restricted isometry with $\delta_{s}(\bar{A}) \leq \delta$.

For random subgaussian matrices this result was proved in [9, 57, by an $\varepsilon$-net argument, where one first checks the deviation inequality $\left|\|A x\|_{2}^{2}-1\right| \leq \delta$ with exponentially high probability for a fixed vector $x$ as in (5.3), and afterwards lets $x$ run over some fine net. For random Fourier matrices the problem is harder. It was first addressed in [17] with a little higher exponent than in (5.4); the exponent 4 was obtained in 64, and it is conjectured that the optimal exponent is 1.

Short Khinchin's inequality and Kashin's subspaces Let $1 \leq p<\infty$. The classical Khinchin's inequality states that there exist constants $A_{p}, B_{p}$ such that for all $x=\left(x_{1}, \ldots, x_{n}\right) \in \mathbb{R}^{n}$

$$
A_{p}\|x\|_{2} \leq\left(\underset{\varepsilon \in\{-1,1\}^{n}}{\operatorname{Ave}}\left|\sum_{j=1}^{n} \varepsilon_{j} x_{j}\right|^{p}\right)^{1 / p} \leq B_{p}\|x\|_{2} .
$$

The average here is taken over all $2^{n}$ possible choices of signs $\varepsilon$ (it is the same as the expectation with respect to independent Bernoulli random variables $\varepsilon_{j}$ ). Since the mid-seventies, the question was around whether Khinchin's inequality holds for averages over some small sets of signs $\varepsilon$. A trivial lower bound follows by a dimension argument: such a set must contain at least $n$ points. Here we shall discuss only the case $p=1$, which is of considerable interest for computer science. This problem can be stated more precisely as follows: as follows:

Given $\delta>0$, find $\alpha(\delta), \beta(\delta)>0$ and construct a set $V \subset\{-1,1\}^{n}$ of cardinality less than $(1+\delta) n$ such that for all $x=\left(x_{1}, \ldots, x_{n}\right) \in \mathbb{R}^{n}$

$$
\alpha(\delta)\|x\|_{2} \leq \underset{\varepsilon \in V}{\operatorname{Ave}}\left|\sum_{j=1}^{n} \varepsilon_{j} x_{j}\right| \leq \beta(\delta)\|x\|_{2} .
$$

The first result in this direction belongs to Schechtman [70 who found an affirmative solution to this problem for $\delta$ greater than some absolute constant. He considered a set $V$ consisting of $N=\lfloor(1+\delta) n\rfloor$ independent random \pm 1 vectors, which can be written as an $N \times n$ random Bernoulli matrix $A$. In the matrix language, the inequality above reads $\alpha(\delta)\|x\|_{2} \leq N^{-1}\|A x\|_{1} \leq \beta(\delta)\|x\|_{2}$ for all $x \in \mathbb{R}^{n}$. This means that one can take

$$
\alpha(\delta)=N^{-1} \inf _{x \in S^{n-1}}\|A x\|_{1}, \quad \beta(\delta)=N^{-1} \sup _{x \in S^{n-1}}\|A x\|_{1} .
$$

These expressions bear a similarity to the smallest and the largest singular values of the matrix $A$. In fact, up to the coefficient $N^{-1}, \beta(\delta)$ is the norm of $A$ considered as a linear operator from $\ell_{2}^{n}$ to $\ell_{1}^{n}$, and $\alpha(\delta)$ is the reciprocal of the norm of its inverse. Schechtman's theorem can now be derived using the $\varepsilon$-net argument.

The case of small $\delta$ is more delicate. For a random $A$, the bound for $\beta(\delta) \leq C$ can be obtained by the $\varepsilon$-net argument as before. However, an attempt to apply 
this argument for $\alpha(\delta)$ runs into to the same problems as for the smallest singular value. For any fixed $\delta>0$ the solution was first obtained first by Johnson and Schechtman [38] who showed that there exists $V$ satisfying (5.5) with $\alpha(\delta)=c^{1 / \delta}$. In [54 this was established for a random set $V$ (or a random matrix $A$ ) with the same bound on $\alpha(\delta)$. Furthermore, the result remains valid even when $\delta$ depends on $n$, as long as $\delta \geq c / \log n$. The proof uses the smallest singular value bound from [53] in a crucial way. The bound on $\alpha(\delta)$ has been further improved in [2], also using the singular value approach. Finally, a theorem in 62 asserts that for a random set $V$ the inequalities (5.5) hold with high probability for

$$
\alpha(\delta)=c \delta^{2}, \quad \beta(\delta)=C
$$

Moreover, the result holds for all $\delta>0$ and $n$, without any restrictions. The proof combines the methods of 63 and a geometric argument based on the structure of a section of the $\ell_{1}^{n}$ ball. The probability estimate of 62 can be further improved if one replaces the small ball probability bound of [63] with that of 65$]$.

The short Khinchin inequality shows also that the $\ell_{1}$ and $\ell_{2}$ norms are equivalent on a random subspace $E:=A \mathbb{R}^{n} \subset \mathbb{R}^{N}$. Indeed, if $A$ is an $N \times n$ random matrix, then with high probability every vector $x \in \mathbb{R}^{n}$ satisfies $\alpha(\delta)\|x\|_{2} \leq$ $N^{-1}\|A x\|_{1} \leq N^{-1 / 2}\|A x\|_{2} \leq C\|x\|_{2}$. The second inequality here is CauchySchwartz, and the third one is the largest singular value bound. Thierefore

$$
C^{-1} \alpha(\delta)\|y\|_{2} \leq N^{-1 / 2}\|y\|_{1} \leq\|y\|_{2} \quad \text { for all } y \in E
$$

Subspaces E possessing property (5.6) are called Kashin's subspaces. The classical Dvoretzky theorem states that a high-dimensional Banach space has a subspace which is close to Euclidean [59. The dimension of such subspace depends on the geometry of the ambient space. Milman proved that such subspaces always exist in dimension $c \log n$, where $n$ is the dimension of the ambient space [58] (see also [59]). For the space $\ell_{1}^{n}$ the situation is much better, and such subspaces exist in dimension $(1-\delta) n$ for any constant $\delta>0$. This was first proved by Kashin [41] also using a random matrix argument. Obviously, as $\delta \rightarrow 0$, the distance between the $\ell_{1}$ and $\ell_{2}$ norms on such subspace grows to $\infty$. The optimal bound for this distance has been found by Garnaev and Gluskin [28] who used subspaces generated by Gaussian random matrices.

Kashin's subspaces turned out to be useful in theoretical computer science, in particular in the nearest neighbor search [36] and in compressed sensing. At present no deterministic construction is known of such subspaces of dimension $n$ proportional to $N$. The result of $[62$ shows that a $\lfloor(1+\delta) n\rfloor \times n$ random Bernoulli matrix defines a Kashin's subspace with $\alpha(\delta)=c \delta^{2}$. A random Bernoulli matrix is computationally easier to implement than a random Gaussian matrix, while the distance between the norms is not much worse than in the optimal case. At the same time, since the subspaces generated by a Bernoulli matrix are spanned by random vertices of the discrete cube, they have relatively simple structure, which is possible to analyze. 


\section{References}

[1] R. Adamczak, A. Litvak, A. Pajor, N. Tomczak-Jaegermann, Quantitative estimates of the convergence of the empirical covariance matrix in log-concave ensembles, J. Amer. Math. Soc. 23 (2010), 535-561.

[2] S. Artstein-Avidan, O. Friedland, V. D. Milman, S. Sodin, Polynomial bounds for large Bernoulli sections of $l_{1}^{N}$, Israel J. Math. 156 (2006), 141-155.

[3] G. Aubrun, A sharp small deviation inequality for the largest eigenvalue of a random matrix, Séminaire de Probabilités XXXVIII, 320-337, Lecture Notes in Math., 1857, Springer, Berlin, 2005.

[4] Z. D. Bai, Circular law, Ann. Probab. 25 (1997), no. 1, 494-529.

[5] Z. D. Bai, Methodologies in spectral analysis of large dimensional random matrices, a review, Statistica Sinica 9 (1999), 611-677

[6] Z. D. Bai, J. Silverstein, Spectral analysis of large dimensional random matrices, 2nd ed., Springer Series in Statistics, Springer, New York, 2010.

[7] Z. D. Bai, J. Silverstein, Y. Q. Yin, A note on the largest eigenvalue of a largedimensional sample covariance matrix, J. Multivariate Anal. 26 (1988), 166-168.

[8] Z. D. Bai,Y. Q. Yin, Limit of the smallest eigenvalue of a large-dimensional sample covariance matrix, Ann. Probab. 21 (1993), 1275-1294.

[9] R. Baraniuk, M. Davenport, R. DeVore, M. Wakin, A simple proof of the restricted isometry property for random matrices, Constr. Approx. 28 (2008), 253-263.

[10] G. Bennett, L. E. Dor, V. Goodman, W. B. Johnson, C. M. Newman, On uncomplemented subspaces of $L_{p}, 1<p<2$, Israel J. Math. 26 (1977), 178-187.

[11] A. C. Berry, The accuracy of the Gaussian approximation to the sum of independent variables, Trans. Amer. Math. Soc., 49 (1941), 122-136.

[12] J. Bourgain, P. Wood, V. Vu, On the singularity probability of random discrete matrices, submitted.

[13] T. Cai, L. Wang, and G. Xu, Shifting Inequality and Recovery of Sparse Signals, IEEE Transactions on Signal Processing, to appear.

[14] E. Candés, Compressive sampling, International Congress of Mathematicians. Vol. III, 1433-1452, Eur. Math. Soc., Zürich, 2006.

[15] E. Candés, The restricted isometry property and its implications for compressed sensing, C. R. Math. Acad. Sci. Paris 346 (2008), 589-592.

[16] E. Candés, T. Tao, Decoding by linear programming, IEEE Trans. Inform. Theory 51 (2005), 4203-4215.

[17] E. Candés, T. Tao, Near-optimal signal recovery from random projections: universal encoding strategies? IEEE Trans. Inform. Theory 52 (2006), 5406-5425.

[18] E. Candés, M. B. Wakin, An Introduction To Compressive Sampling, IEEE Signal Processing Magazine, V.21, March 2008.

[19] K. R. Davidson, S. J. Szarek, Local operator theory, random matrices and Banach spaces. Handbook of the geometry of Banach spaces, Vol. I, 317-366, North-Holland, Amsterdam, 2001.

[20] A. Edelman, Eigenvalues and condition numbers of random matrices, SIAM J. Matrix Anal. Appl. 9 (1988), 543-560 
[21] P. Erdös, On a lemma of Littlewood and Offord, Bull. Amer. Math. Soc. 51 (1945), 898-902

[22] P. Erdös, Extremal problems in number theory, 1965 Proc. Sympos. Pure Math., Vol. VIII, pp.181-189 AMS, Providence, R.I.

[23] C. G. Esseen, Fourier analysis of distribution functions. A mathematical study of the laplace Gaussian law, Acta Math. 77 (1945), 1-125.

[24] C. G. Esseen, On the Kolmogorov-Rogozin inequality for the concentration function, Z. Wahrscheinlichkeitstheorie und Verw. Gebiete 5 (1966), 210-216.

[25] O. Feldheim, S. Sodin, A universality result for the smallest eigenvalues of certain sample covariance matrices, Geometric and Functional Analysis, to appear

[26] P. Frankl, Z. Füredi, Solution of the Littlewood-Offord problem in high dimensions, Ann. of Math. (2) 128 (1988), 259-270.

[27] O. Friedland, S. Sodin, Bounds on the concentration function in terms of the Diophantine approximation, C. R. Math. Acad. Sci. Paris 345 (2007), 513-518.

[28] A. Garnaev, E. Gluskin, The widths of a Euclidean ball, Soviet Math. Dokl. 30 (1984), 200-204.

[29] S. Geman, A limit theorem for the norm of random matrices, Ann. Probab. 8 (1980), $252-261$.

[30] V. L. Girko, The circular law, Theory Probab. Appl. 29 (1984), no. 4, 694-706.

[31] Y. Gordon, On Dvoretzky's theorem and extensions of Slepian's lemma, Israel seminar on geometrical aspects of functional analysis (1983/84), II, Tel Aviv Univ., Tel Aviv, 1984.

[32] Y. Gordon, Some inequalities for Gaussian processes and applications, Israel J. Math. 50 (1985), 265-289.

[33] Y. Gordon, Majorization of Gaussian processes and geometric applications, Probab. Theory Related Fields 91 (1992), 251-267.

[34] F. Götze, A. Tikhomirov, On the Circular Law, arXiv:math/0702386.

[35] F. Götze, A. Tikhomirov, The Circular Law for Random Matrices, arXiv:0709.3995 , to appear in Ann. Prob.

[36] P. Indyk, Dimensionality reduction techniques for proximity problems, Proceedings of the Ninth ACM-SIAM Symposium on Discrete Algorithms, 2000.

[37] K. Johansson, Shape fluctuations and random matrices, Comm. Math. Phys. 209 (2000), 437-476.

[38] W. B. Johnson, G. Schechtman, Very tight embeddings of subspaces of $L_{p}, 1 \leq p<2$, into $l_{p}^{n}$, Geom. Funct. Anal. 13 (2003), no. 4, 845-851.

[39] I. M. Johnstone, On the distribution of the largest eigenvalue in principal components analysis, Ann. Statist. 29 (2001), 295-327.

[40] G. Halász, Estimates for the concentration function of combinatorial number theory and probability, Periodica Mathematica Hungarica 8 (1977), 197-211

[41] B. Kashin, Section of some finite-dimensional sets and classes of smooth functions (in Russian) Izv. Acad. Nauk. SSSR 41 (1977) 334-351.

[42] A. Kolmogorov, Sur les propriétés des fonctions de concentrations de M. P. Lévy, Ann. Inst. H. Poincaré 16 (1958), 27-34. 
[43] J. Kahn, J. Komlós, E. Szemerédi, On the probability that a random \pm 1 -matrix is singular, J. Amer. Math. Soc. 8 (1995), 223-240.

[44] J. Komlós, On the determinant of $(0,1)$ matrices, Studia Sci. Math. Hungar. 2 (1967), $7-22$.

[45] J. Komlós, On the determinant of random matrices, Studia Sci. Math. Hungar. 3 (1968), 387-399.

[46] R. Latala, Some estimates of norms of random matrices, Proc. Amer. Math. Soc. 133 (2005), 1273-1282.

[47] M. Ledoux, The concentration of measure phenomenon. Mathematical Surveys and Monographs, 89. American Mathematical Society, Providence, RI, 2001.

[48] M. Ledoux, Deviation inequalities on largest eigenvalues, Geometric aspects of functional analysis, 167-219, Lecture Notes in Math., 1910, Springer, Berlin, 2007.

[49] M. Ledoux, M. Talagrand, Probability in Banach spaces. Isoperimetry and processes. Ergebnisse der Mathematik und ihrer Grenzgebiete (3), 23. Springer-Verlag, Berlin, 1991.

[50] P. Lévy, Théorie de l'addition des variables aléatoires, Gauthier-Villars, 1937.

[51] W. V. Li, Q.-M. Shao, Gaussian processes: inequalities, small ball probabilities and applications. Stochastic processes: theory and methods, 533-597, Handbook of Statistics, 19, North-Holland, Amsterdam, 2001.

[52] J. E. Littlewood, A. C. Offord, On the number of real roots of a random algebraic equation. III. Rec. Math. [Mat. Sbornik] N.S. 12 (54), (1943), 277-286

[53] A. Litvak, A. Pajor, M. Rudelson, N. Tomczak-Jaegermann, Smallest singular value of random matrices and geometry of random polytopes, Adv. Math. 195 (2005), 491523.

[54] A. E. Litvak, A. Pajor, M. Rudelson, N. Tomczak-Jaegermann, R. Vershynin, Euclidean embeddings in spaces of finite volume ratio via random matrices, J. Reine Angew. Math. 589 (2005), 1-19.

[55] V. A. Marchenko, L. A. Pastur, The distribution of eigenvalues in certain sets of random matrices, Mat. Sb., 72 (1967), 507-536.

[56] M. L. Mehta, Random matrices and the statistical theory of energy levels. Academic Press, New York-London 1967.

[57] S. Mendelson, A. Pajor, N. Tomczak-Jaegermann, Uniform uncertainty principle for Bernoulli and subgaussian ensembles, Constr. Approx. 28 (2008), 277-289.

[58] V. Milman, A new proof of the theorem of A. Dvoretzky on sections of convex bodies, Funct. Anal. Appl. 5 (1971), 28-37.

[59] V. D. Milman, G. Schechtman, Asymptotic theory of finite-dimensional normed spaces. With an appendix by M. Gromov. Lecture Notes in Math. 1200, SpringerVerlag, Berlin 1986.

[60] L. A Pastur, On the spectrum of random matrices, Teoret. Mat. Fiz. 10 (1973), 102-112.

[61] V. V. Petrov, Sums of independent random variables. Ergebnisse der Mathematik und ihrer Grenzgebiete, Band 82. Springer-Verlag, New York-Heidelberg, 1975.

[62] M. Rudelson, Lower estimates for the singular values of random matrices, C. R. Math. Acad. Sci. Paris 342 (2006), 247-252. 
[63] M. Rudelson, Invertibility of random matrices: norm of the inverse, Annals of Mathematics 168 (2008), 575-600.

[64] M. Rudelson, R. Vershynin, On sparse reconstruction from Fourier and Gaussian measurements, Communications on Pure and Applied Mathematics 61 (2008), 10251045.

[65] M. Rudelson, R. Vershynin, The Littlewood-Offord problem and invertibility of random matrices, Adv. Math. 218 (2008), 600-633.

[66] M. Rudelson, R. Vershynin, The least singular value of a random square matrix is $O\left(n^{-1 / 2}\right)$, Comptes rendus de l'Académie des sciences - Mathématique 346 (2008), 893-896.

[67] M. Rudelson, R. Vershynin, Smallest singular value of a random rectangular matrix, Comm. Pure Appl. Math. 62 (2009), 1707-1739.

[68] A. Sankar, D. A. Spielman, S.-H. Teng, Smoothed analysis of the condition numbers and growth factors of matrices, SIAM J. Matrix Anal. Appl. 28 (2006), 446-476.

[69] A. Sárközy, E. Szeméredi, Über ein Problem von Erdös und Moser, Acta Arithmetica 11 (1965), 205-208

[70] G. Schechtman, Random embeddings of Euclidean spaces in sequence spaces, Israel Journal of Mathematics 40, No. 2, 1981, $187 ? 192$.

[71] J. Silverstein, The smallest eigenvalue of a large-dimensional Wishart matrix, Ann. Probab. 13 (1985), 1364-1368.

[72] D. Slepian, The one-sided barrier problem for Gaussian noise, Bell System Tech. J. 41 (1962), 463-501.

[73] S. Smale, On the efficiency of algorithms of analysis, Bull. Amer. Math. Soc. (N.S.) 13 (1985), 87-121

[74] A. Soshnikov, A note on universality of the distribution of the largest eigenvalues in certain sample covariance matrices, J. Statist. Phys. 108 (2002), 1033-1056.

[75] D. Spielman, S.-H. Teng, Smoothed analysis of algorithms. Proceedings of the International Congress of Mathematicians, Vol. I (Beijing, 2002), 597-606, Higher Ed. Press, Beijing, 2002

[76] M. Talagrand, Concentration of measure and isoperimetric inequalities in product spaces, Inst. Hautes Études Sci. Publ. Math. No. 81 (1995), 73-205.

[77] M. Talagrand, A new look at independence, Ann. Probab. 24 (1996), 1-34.

[78] T. Tao, V. Vu, On random \pm 1 matrices: singularity and determinant, Random Structures and Algorithms 28 (2006), 1-23.

[79] S. Szarek, Condition numbers of random matrices, J. Complexity 7 (1991), 131-149.

[80] T. Tao, V. Vu, Additive combinatorics. Cambridge Studies in Advanced Mathematics, 105. Cambridge University Press, Cambridge, 2006.

[81] T. Tao, V. Vu, On the singularity probability of random Bernoulli matrices, J. Amer. Math. Soc. 20 (2007), 603?628.

[82] T. Tao, V. Vu, Random matrices: the circular law, Commun. Contemp. Math. 10 (2008), no. 2, 261-307.

[83] T. Tao, V. Vu, Inverse Littlewood-Offord theorems and the condition number of random discrete matrices, Annals of Math. 169 (2009), 595-632. 
[84] T. Tao, V. Vu, From the Littlewood-Offord problem to the circular law: universality of the spectral distribution of random matrices, Bull. Amer. Math. Soc. 46 (2009), 377-396.

[85] T. Tao, V. Vu (with appendix by M. Krishnapur), Random matrices: Universality of ESDs and the circular law, arXiv:0808.4898, to appear in Ann. Prob.

[86] T. Tao, V. Vu, A sharp inverse Littlewood-Offord theorem, Random Structures and Algorithms, to appear

[87] T. Tao, V. Vu, Random matrices: the distribution of smallest singular values, Geom. Funct. Anal., to appear

[88] T. Tao, V. Vu, The Littlewood-Offord problem in high dimensions and a conjecture of Frankl and Füredi, preprint

[89] C. Tracy, H. Widom, Level-Spacing Distributions and the Airy Kernel, Comm. Math. Phys. 159 (1994), 151-174.

[90] C. Tracy, H. Widom, On orthogonal and symplectic matrix ensembles, Comm. Math. Phys. 177 (1996), no. 3, 727?-754.

[91] C. Tracy, H. Widom, Distribution functions for largest eigenvalues and their applications, Proceedings of the International Congress of Mathematicians, Vol. I (Beijing, 2002), 587-596, Higher Ed. Press, Beijing, 2002.

[92] J. von Neumann, H. H. Goldstine, Numerical inverting of matrices of high order, Bull. Amer. Math. Soc. 53 (1947), 1021-1099.

[93] R. Vershynin, Spectral norm of products of random and deterministic matrices, Probability Theory and Related Fields, DOI: 10.1007/s00440-010-0281-z.

[94] R. Vershynin, How close is the sample covariance matrix to the actual covariance matrix? preprint.

[95] K. W. Wachter, Strong limits of random matrix spectra for sample covariance matrices of independent elements, Annals of Probability 6 (1978), 1-18.

[96] P. Wigner, On the distribution of the roots of certain symmetric matrices, Annals of Mathematics 67 (1958), 325-327.

[97] Y. Q. Yin, Z. D. Bai, P. R. Krishnaiah, On the limit of the largest eigenvalue of the large-dimensional sample covariance matrix, Probab. Theory Related Fields 78 (1988), 509-521.

Department of Mathematics University of Missouri-Columbia Columbia, Missouri, U.S.A.

E-mail: rudelsonm@missouri.edu

Department of Mathematics University of Michigan

Ann Arbor, Michigan, U.S.A.

E-mail: romanv@umich.edu 